\title{
Disordered Quantum smectics
}

\author{
E. Orignac \\ Laboratoire de Physique Théorique de l'École Normale Supérieure \\ CNRS-UMR8549 24,Rue Lhomond 75231 Paris Cedex 05 France \\ R. Chitra \\ Laboratoire de Physique Théorique des Liquides CNRS-UMR7600 \\ Université Pierre et Marie Curie, 4 Place Jussieu 75252 Paris Cedex 05 France
}

\begin{abstract}
We study the impurity pinning of the Quantum Hall (QH) smectic state arising in two dimensional electron systems in high Landau levels. We use replicas and a Gaussian Variational method to deal with the disorder. The pinned quantum smectic exhibits very anisotropic behaviour, with density correlations along the direction of the stripes manifesting a Bragg-Glass type behaviour i.e., quasi long range order whereas those in the transverse direction are infra red divergent. We calculate the dynamical conductivity $\sigma_{x x}(\mathbf{q}, \omega)$ along the stripe direction and find a $\mathbf{q}$ dependent pinning peak.
\end{abstract}

Two dimensional electron gases subjected to perpendicular magnetic fields are known to exhibit a plethora of quantum phases. The Integer and Fractional Quantum Hall Effects, Wigner Crystallization etc... are but some of the phases observed experimentally [1]. Most of these phases appear in strong magnetic fields where the electrons occupy the lowest Landau level. On the other hand, certain anisotropic phases have been observed in the 2DEG when the electrons occupy reasonably high Landau levels $(\nu=9 / 2)$ [2]. This seemed to be in accord with Hartree-Fock calculations [3], which predicted the existence of new phases such as bubble phases and charge density waves (CDW) for intermediate to weak magnetic fields. Consequently, this experimentally seen anisotropic phase was thought to be a simple CDW where the electron density is modulated in only one direction resulting in a ground state where electron rich stripes alternate with hole rich stripes. This idea offered a simple explanation of the anisotropic resistivities measured in experiments. Since the CDW is expected to be pinned by the incipient disorder it implies the existence of a finite threshold electric field for the CDW to slide. However, though there was a substantial increase in the differential conductivity, no well defined threshold was seen in the $I-V$ characteristics of the anisotropic phase 2]. This cast serious doubts on the stability of the CDW phase predicted by Hartree Fock theory with respect to quantum and thermal fluctuations. It is now believed that rather than a CDW or stripe crystal, the correct stable ground state is a fluctuating state analogous to a smectic A liquid crystal[ $\mid$.

In the absence of impurities, the properties of this smectic liquid crystal have been thoroughly investigated [5, 6, 7, 8]. In the presence of impurities, the theoretical situation is much less clear. Conflicting results on the stability of the smectic phase in the presence of disorder have been obtained in [6] and in [9]. In Ref. [10], the effect of disorder on the classical analog of the quantum Hall smectic was studied using the renormalization group method developped in Ref. 11 for the conventional classical smectics. However, the coupling to disorder used in [9, 10] is chosen in analogy with the conventional smectic and is not the pertinent one for the Quantum Hall smectic.

In the present letter, we revisit the problem of impurity pinning of the quantum smectic. We derive the appropriate coupling to impurities and tackle the effect of disorder non-perturbatively, using a powerful variational method 12] that has previously been applied successfully to various quantum elastic systems such as dirty Luttinger liquids 13] and Wigner crystals [14]. Our model differs from that of [9, 10] in the way disorder couples to the system and this difference is crucial in obtaining the transport and correlations in the pinned Quantum Hall smectic.

In the smectic phase, the ground state of the two dimensional electron gas is characterized by alternating stripes of filling factors $\nu$ and $\nu+1$ with a period $\lambda$ which is of the order of a few cyclotron lengths. The smectic layers are taken to be parallel to the $x$ direction in the $x-y$ plane. These fluctuating stripes interact with each other on short scales and are subject to residual Coulomb long range forces on large scales. Starting from the theory of [6] and using a Hamiltonian framework, we have obtained the following effective action [15] to describe the low energy properties of the quantum Hall smectic

$$
S=\frac{1}{\beta} \sum_{n} \int \frac{d^{2} \mathbf{q}}{(2 \pi)^{2}} G_{0}^{-1}\left(\mathbf{q}, \omega_{n}\right)\left|\phi\left(\mathbf{q}, \omega_{n}\right)\right|^{2},
$$

where $\phi$ is the coarse-grained local width of a stripe and

$$
G_{0}^{-1}\left(\mathbf{q}, \omega_{n}\right)=\frac{\omega_{n}^{2}}{Q q_{x}^{4}+\frac{\kappa \perp}{\left|q_{x}\right|} q_{y}^{2}}+\kappa_{\|}\left|q_{x}\right| .
$$

The coefficients $Q, \kappa_{\|}$and $\kappa_{\perp}$ can be obtained from the Hartree-Fock energy calculations on the original electronic problem [7, 16]. $\omega_{n}=2 \pi n / \beta$ are the standard bosonic Matsubara frequencies with $\beta=1 / T$. Henceforth, we set $\hbar=1$. This action is the same as that 
derived from a Chern-Simons description of the Hall system presented in [5]. In the rest of the Letter, we use the same notations as in [5].

In the absence of disorder, the above effective action leads to the following correlation functions 5 for the stripe width, $C_{\phi}(x, 0,0) \sim 1 /(2 \lambda)^{2}\left(\kappa_{\perp} / \kappa_{\|}\right)^{1 / 2} \ln (|x| / \alpha)$ where $\alpha$ is a short distance cutoff and $C_{\phi}(x, y, \tau)=$ $\left\langle(\phi(x, y, \tau)-\phi(0,0,0))^{2} / 2\right\rangle$. Correlation in the direction perpendicular to the stripes are infrared divergent. These correlations are divergent even in the case of short ranged forces. Inter-stripes process such as tunneling and backscattering may render the stripes more rigid in the perpendicular direction resulting in finite correlations. It has however, been shown that coulomb interactions do not trigger any instability towards Wigner

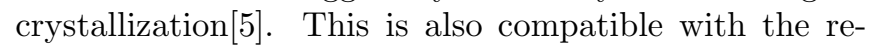
sults of Ref. 16 where time dependent Hartree Fock calculations indicate that the smectic state is indeed stable to quantum fluctuations. These correlation functions are clearly anisotropic. Since the density correlation function is related to the width correlation by the equation $C_{\rho}(x, y, 0)=\exp -2 C_{\phi}(x, y, 0)$ 络, 15], we see that the density fluctuations of the quantum smectic parallel to the stripe are indeed stable to quantum fluctuations. Within the present formalism, the stripe displacement and width being canonically conjugate variables [15, correlations of the stripe displacement can also be calculated.

Interaction of the system with impurities correspond to backward scattering of chiral electrons or holes from one edge of the QH stripe to another edge of opposite chirality. Disorder induces both intra-stripe and inter-stripe backward scattering processes. We shall assume in the following that the intra-stripe processes are the dominant ones and neglect inter-stripe processes. This approximation is valid for inter-stripe distances much larger than the stripe width. In the continuum limit, the coupling of disorder to the electron density can be written using the expression for the edge fermion creation and annihilation operators [6]. In terms of the field $\phi$, the combined action reads:

$$
S=S_{0}+\int d^{2} \mathbf{r} d \tau\left[\xi(\mathbf{r}) e^{2 i \phi(\mathbf{r}, \tau)}+\text { H. c. }\right]
$$

For tractability, we assume a Gaussian distributed disorder, $\overline{\xi(\mathbf{r}) \xi^{*}\left(\mathbf{r}^{\prime}\right)}=\frac{\Delta}{4} \delta\left(\mathbf{r}-\mathbf{r}^{\prime}\right)$ where $\Delta$ is a measure of the strength of disorder. In Ref. [6], it has been shown that this type of disorder is a relevant perturbation. In Refs. [9, 10], it was incorrectly assumed that disorder coupled to the average displacement of the stripe instead of the electron density. In physical terms, the effect of im- purities is not to deform the stripe structure by coupling to the position of the stripe as in the classical case, but rather to cut the stripes into disconnected pieces thus suppressing transport along the stripe direction. This difference of coupling is the reason for the discrepancies of [6] and [9].

Before, we proceed with the full treatment of the disordered Hall smectic, we first look at the equivalent of the Larkin random force model 17] for a $d$-dimensional quantum smectic (consisting of $d-1$ dimensional stripes stacked along the $y$ axis) to estimate the relevance of disorder. Within the Larkin approximation, where the disorder couples directly to the width $\phi$, the width correlation function is given by

$C_{\phi}(x, y, 0)=\frac{\Delta}{(2 \pi)^{d}} \int_{0}^{\infty} d^{d-1} q_{x} \int_{-\frac{\pi}{\lambda}}^{\frac{\pi}{\lambda}} d q_{y} \frac{\left(1-\cos \left(\mathbf{q}_{x} \cdot \mathbf{x}+q_{y} y\right)\right)}{\kappa_{\|}^{2} q_{x}^{2}}$

which yields the following correlations: $C_{\phi}(x, 0,0) \sim$ $\frac{\Delta}{\kappa_{\|}} x^{3-d}$, with fluctuations in the perpendicular direction remaining divergent. From this analysis, smectic order along the stripes is very sensitive to dimensionality. The upper critical dimension above which disorder is irrelevant is $d=3$ and the lower critical dimension is $d=2$ below which, quantum and thermal fluctuations effectively wipe out the disorder. Thus, the quantum smectic is affected by impurities in a strongly anisotropic manner. Note that here we are dealing exclusively with the effect of disorder on the correlations of the stripe width and the conclusions do not hold for the stripe displacement correlations. However, a similar analysis can be done to study the effect of disorder on the correlation of the stripe displacements 15].

The Larkin analysis can also be used to estimate the length scale $R_{a}$ over which the system disorders. In the present problem, since the field $\phi$ is like a phase variable, $R_{a}$ is defined as the length scale over which the relative phase changes by $2 \pi$ i.e., $C_{\phi}\left(R_{a}, 0,0\right)=(2 \pi)^{2}$. We find that in $d=2$,

$$
R_{a} \simeq \frac{4 \pi^{2} \kappa_{\|}^{2} \lambda}{\Delta}
$$

To go beyond the Larkin approximation, we now use replicas and the Gaussian variational method to study the long wavelength properties of the Quantum Hall smectic with both Coulomb repulsion and impurities described by the action (3). Introducing replicas and averaging over disorder, we obtain the following effective action, 


$$
S_{\mathrm{r}}=\sum_{a} S_{0}\left[\phi_{a}\right]-\frac{\Delta}{4} \sum_{a b} \int d x d y d \tau d \tau^{\prime} \cos 2\left[\phi_{a}(x, y, \tau)-\phi_{b}\left(x, y, \tau^{\prime}\right)\right]
$$

To deal with this effective action where the disorder manifests itself as an interaction non-local in time, we use the Feynman variational principle. We introduce the following quadratic variational action:

$$
S_{\text {trial }}=\frac{1}{2 \beta} \sum_{n} \int \frac{d^{2} \mathbf{q}}{(2 \pi)^{2}} \sum_{a, b} G_{a b}\left(\mathbf{q}, i \omega_{n}\right)\left|\phi\left(\mathbf{q}, i \omega_{n}\right)\right|^{2}
$$

where the trial Green's functions $G_{a b}$ are parameterized as $G_{a b}^{-1}\left(\mathbf{q}, i \omega_{n}\right)=G_{0}^{-1}\left(\mathbf{q}, i \omega_{n}\right) \delta_{a b}-\sigma_{a b}\left(\mathbf{q}, i \omega_{n}\right)$ and $\sigma_{a b}$ are the disorder induced replica self energies. Since the disorder is local, the $\sigma_{a b}$ are independent of the momentum q. These self-energies are determined in a selfconsistent manner by extremizing the variational free energy $F_{\text {var }}=F_{\text {trial }}+\frac{1}{\beta}\left\langle S_{\mathrm{r}}-S_{\text {trial }}\right\rangle_{S_{\text {trial }}}$ with respect to the $G_{a b}$. There are two generic solutions to these saddle point equations, one with replica symmetry where $\sigma_{a \neq b}=\sigma, \forall a, b$ and the other with broken replica symmetry. For the quantum system studied in this letter the appropriate low temperature solution is the one with replica symmetry breaking [13].
Using the machinery developed in [12] and proceeding along the lines of 13, we find that a full treatment of the disorder in a $d$ dimensional smectic, confirms the Larkin analysis predictions that $d=3(d=2)$ are the upper (lower) critical dimensions for disorder [15]. Moreover, since $d=2$ is the lower critical dimension, the solution to the problem of the Hall smectic considered here, has one-step replica symmetry breaking. For the one-step solution, the connected part of the Green's function defined as $G_{c}^{-1}=\sum_{b} G_{a b}^{-1}$ has the following structure:

$$
G_{c}^{-1}\left(q, i \omega_{n}\right)=G_{0}^{-1}\left(q, i \omega_{n}\right)+I\left(i \omega_{n}\right)+\Sigma\left(1-\delta_{\omega_{n}, 0}\right) .
$$

$\Sigma$ which is independent of both $q$ and the frequency, is a mass term describing impurity pinning while $I\left(i \omega_{n}\right)$ describes the dissipation induced by disorder. The saddlepoint equations can be reduced 15 to self-consistent equations $\Sigma$ and $I\left(i \omega_{n}\right)$. We find that $\Sigma=\kappa_{\|} R_{a}^{-1}$ with $R_{a}$ given by (5) 15. $I$ is self consistently determined in the semi-classical limit [13] by the equation

$$
I\left(i \omega_{n}\right)=\frac{\lambda \kappa_{\|} \Sigma}{4 \pi} \int_{-\infty}^{\infty} d q_{x} \int_{-\frac{\pi}{\lambda}}^{\frac{\pi}{\lambda}} d q_{y}\left[\frac{1}{\kappa_{\|}\left|q_{x}\right|+\Sigma}-\frac{Q q_{x}^{4}+\frac{\kappa_{\perp}}{\left|q_{x}\right|} q_{y}^{2}}{\omega_{n}^{2}+\left(\kappa_{\|}\left|q_{x}\right|+\Sigma+I\left(i \omega_{n}\right)\right)\left(Q q_{x}^{4}+\frac{\kappa_{\perp}}{\left|q_{x}\right|} q_{y}^{2}\right)}\right]
$$

$I$ determines all dynamical properties and for $\omega_{n} \rightarrow 0$ : $I\left(i \omega_{n}\right) \sim \omega_{n}^{\frac{7}{8}}$. For arbitrary frequencies, the equation (9) can be continued to real frequencies and solved numerically.

We now study the effect of disorder on various physical properties at zero temperature. Using the results of [13], the disorder averaged equal-time width correlation function along the stripe, is obtained as:

$$
\tilde{C}_{\phi}(x, 0,0) \simeq \frac{1}{2 \pi \lambda \kappa_{\|}} \log \left(\frac{x}{R_{a}}\right)
$$

The system retains logarithmic correlations along the stripe directions, albeit with a different prefactor, which is now independent of $\kappa_{\perp}$. Also, the short distance cutoff $\alpha$ is replaced by the lengthscale $R_{a}$. Correlations in the perpendicular direction remain infra-red divergent as before. Logarithmic increase of correlations in disordered system are characteristic of Bragg-Glass phases where density correlations have quasi-long range order. Similar Bragg-glass phases have been previously obtained in Wigner crystals 14 and vortex lattices 13 . Although these density fluctuations are what is measured by diffraction, the true order of the stripe phase is measured by correlation of the displacement. Since the displacement and width fluctuations are canonically conjugate, pinning of the width fluctuations would imply that the stripe structure becomes strongly disordered by wild fluctuations in the displacement. Note that our results differ greatly from that of 10 where logarithmic correlations were obtained for the stripe displacements in both parallel and perpendicular directions. This can be attributed to the fact that in our case disorder couples to the density whereas in Ref. [10], disorder couples to the stripe displacement and the all important quantum fluctuations have been neglected.

Although disorder does not modify strongly the static correlations of the system, it modifies the transport properties in a nontrivial manner. To discuss the transport 
properties, we need to calculate the conductivity tensor $\sigma_{i j}(\mathbf{q}, \omega)=\frac{i}{\omega}\left\langle J_{i}(\mathbf{q}, \omega) J_{j}(-\mathbf{q},-\omega)\right\rangle$. where $\omega$ is the frequency. Clearly, in such an anisotropic state the conductivities parallel and perpendicular to the stripes are unequal. In addition, due to the presence of an external magnetic field, there is the Hall conductivity. Within our approach, we can only access the diagonal component $\sigma_{x x}$ of the conductivity tensor. To obtain the current $J_{x}$ along the $x$ direction, we use the equation of continuity which for for $q_{y}=0$ leads to the expression $q_{x} J_{x}\left(q_{x}, q_{y}=0, \omega\right)=\omega \rho\left(q_{x}, q_{y}=0, \omega\right)$ where the density $\rho\left(q_{x}, q_{y}=0, \omega\right)=i q_{x} \phi\left(q_{x}, q_{y}=0, \omega\right)$. Using this expression for the current, and analytically continuing the Matsubara frequencies to real frequencies $\left(i \omega_{n} \rightarrow \omega+i \epsilon\right)$ in (8), the conductivity along the $x$ direction is given by

$$
\sigma_{x x}\left(q_{x}, q_{y}=0, \omega\right)=C i \omega \frac{Q q_{x}^{4}}{\omega^{2}-\left(\kappa_{\|}\left|q_{x}\right|+\Sigma+I\right) Q q_{x}^{4}}
$$

In the clean system, $\sigma_{x x}=Q q_{x}^{4} \delta\left(\omega-\sqrt{\kappa_{\|} Q}\left|q_{x}\right|^{5 / 2}\right)$. We see that there is no Drude peak for zero momentum but a $\delta$ - function peak in the conductivity at a finite frequency $\left.\omega_{0}\left(q_{x}\right)=\sqrt{\kappa_{\|} Q}\left|q_{x}\right|^{5 / 2}\right)$. In the presence of disorder, akin to the clean system, the zero temperature $\mathrm{dc}$ conductivity parallel to the stripes of the smectic vanishes for all $q_{x}$. From (11), we see that the dynamical conductivity is characterized by a pinning peak at nonzero frequency. Neglecting the dissipative term $I(\omega)$, we find a momentum dependent pinning frequency given by

$$
\omega_{p}=q_{x}^{2} \sqrt{Q \Sigma+Q \kappa_{\|}\left|q_{x}\right|} \simeq \sqrt{Q \Sigma q_{x}^{4}+\omega_{0}^{2}}
$$

For $\kappa_{\|}\left|q_{x}\right| \ll \Sigma, \omega_{p}=\sqrt{Q \Sigma} q_{x}^{2}$. In other disordered systems, the pinning frequency is basically fixed by $\Sigma$ and any small momentum dependence is just a shift in $\omega_{p}$. However, in the case of the smectic, due to the $q_{x}$ dependent pre-factor, $\omega_{p}$ can be made as small as possible by changing $q_{x}$. The effect of the dissipative term $I\left(i \omega_{n}\right)$ is to broaden the delta function at $\omega_{p}$ into a peak in conductivity. This peak is asymmetric as in various other quantum disordered systems 14. Finally, for small $q_{x}$ and $\omega$, such that $Q \Sigma q_{x}^{4} \gg \omega^{2}$, we obtain $\sigma_{x x}\left(q_{x}, \omega\right) \simeq \omega^{\frac{15}{8}} / \Sigma^{2}$. Compared to other disordered systems where the low frequency $q=0$ conductivity is quadratic in $\omega$, here due to the complicated scaling relations between $q_{x}$ and $\omega$, we find an exponent which is slightly sub-quadratic. A plot of the conductivity as a function of $q_{x}$ and $\omega$ is on Fig. 1. These $q$ dependent conductivities can be measured using the meander line techniques previously used to measure the microwave conductivity in Wigner crystals. To summarize, we see that disorder indeed has a big effect on ac conductivity.

Another quantity of interest is the threshold electric field for depinning of the smectic. Extending the LarkinOvchinnikov [18 arguments to the present case, it is reasonable to assume that since the system is pinned in the $x$ direction, the threshold field for depinning along this direction is given by $E_{T x}=\kappa_{\|} R_{a}^{-1} l$ where $l$ is the average width of a stripe. If the $y$ direction was free to slide, the pinning would imply a strong non-linearity in the $\mathrm{I}-\mathrm{V}$ characteristics for an applied field in any arbitrary direction. Nonetheless, the result that the width fluctuations remain divergent along the $y$ - direction does not necessarily imply that there is no threshold field for depinning in this direction. The behaviour of the conductivity and threshold in this direction will be dictated by processes which facilitate the hopping and tunneling of electrons between the stripes. Even in the absence of disorder, the conductivity along the $y$ - direction is through inter-stripe tunneling events. These are not included in our approach and depending on these processes, $\sigma_{y y}(q=0, \omega=0)$ can be zero or finite. These issues are beyond the scope of the paper and will be addressed in [15.

To conclude, we have analyzed the pinning of quantum Hall stripes by impurities using the Gaussian variational method. We find that at $T=0$, the system is pinned and insulating along the stripe direction and density fluctuations exhibit quasi-long range order akin to Bragg glasses. Moreover, the dynamical conductivity in the parallel direction is strongly wave-vector dependent. It would also be of interest to perform a complementary Functional Renormalization Group study of the QH stripes. To calculate $\sigma_{x y}$ and $\sigma_{y y}$ and make a full comparison with experiments, one necessarily has to include processes which enable electrons to coherently hop between stripes. This, however, is beyond the realm of the Gaussian Variational Method.

We thank T. Giamarchi for discussions and for a careful reading of the manuscript.

[1] T. Chakraborty and P. Pietiläinen, The Quantum Hall Effects (Springer, Berlin, 1994).

[2] M. P. Lilly et al., Phys. Rev. Lett. 82, 394 (1999); M. P. Lilly et al., Phys. Rev. Lett. 83, 824 (1999); K. B. Cooper et al., Phys. Rev. B 60, R11285 (1999).

[3] H. Fukuyama, P. Platzman, and P. W. Anderson, Phys. Rev. B 19, 5211 (1979); R. Moessner and J. T. Chalker, Phys. Rev. B 54, 5006 (1996); A. A. Koulakov, M. M. Fogler, and B. I. Shklovskii, Phys. Rev. Lett. 76, 499 (1996).

[4] E. Fradkin and S. A. Kivelson, Phys. Rev. B 59, 8065 (1999).

[5] D. G. Barci, E. Fradkin, S. A. Kivelson, and V. Oganesyan, cond-mat/0105448 (unpublished).

[6] A. H. MacDonald and M. P. A. Fisher, Phys. Rev. B 61, 5724 (2000).

[7] A. Lopatnikova, S. H. Simon, B. I. Halperin, and X.-G. Wen, Phys. Rev. B 64, 155301 (2001); C. Wexler and A. T. Dorsey, Phys. Rev. B 64, 115312 (2001).

[8] M. M. Fogler and V. M. Vinokur, Phys. Rev. Lett. 84, 5828 (2000). 


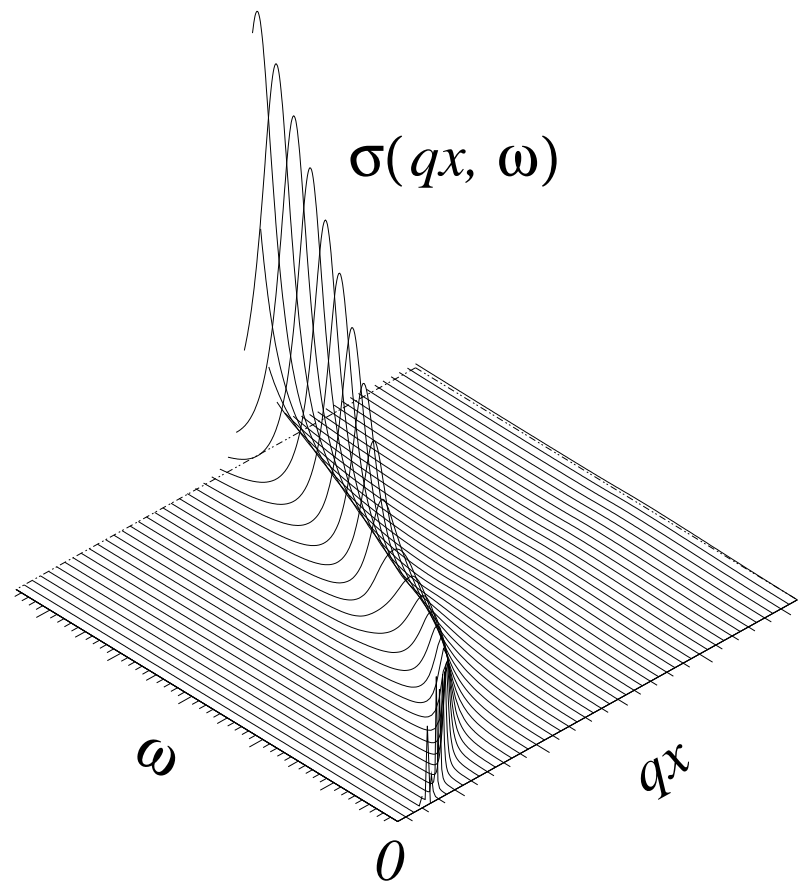

FIG. 1: The conductivity $\sigma_{x x}\left(q_{x}, \omega\right)$ (measured in arbitrary units) along the stripes in the disordered quantum smectic.
[9] H. Yi, H. A. Fertig, and R. Côté, Phys. Rev. Lett. 85, 4156 (2000).

[10] S. Scheidl and F. von Oppen, Europhys. Lett. 55, 260 (2001).

[11] L. Radzihovsky and J. Toner, Phys. Rev. B 60, 206 (1999), cond-mat/9811105.

[12] M. Mézard and G. Parisi, J. de Phys. I 1, 809 (1991).

[13] T. Giamarchi and P. Le Doussal, Phys. Rev. B 52, 1242 (1995); ibid. 53, 15206 (1996).

[14] R. Chitra, T. Giamarchi, and P. Le Doussal, Phys. Rev. Lett. 80, 3827 (1998); Phys. Rev. B 65, 035312 (2002).

[15] R. Chitra and E. Orignac, article in preparation (unpublished).

[16] R. Côté and H. A. Fertig, Phys. Rev. B 62, 1993 (2000).

[17] A. I. Larkin, Sov. Phys. JETP 31, 784 (1970).

[18] A. I. Larkin and Y. N. Ovchinnikov, J. Low. Temp. Phys. 34409 (1979). 